\title{
Performance Analysis of OSTBC Transmission in Lossy Forward MIMO Relay Networks
}

\author{
Jiguang He, Valtteri Tervo, Shen Qian, Markku Juntti, and Tad Matsumoto
}

\begin{abstract}
We analyze the outage probability for the orthogonal space-time block code based multiple-input multiple-output (MIMO) relay networks, composed of one source, one relay, and a single destination. The relay forwards the decoded and interleaved information sequence even though the information part may contain error(s), according to the lossy forward strategy. In spatially independent MIMO channels, we find that the diversity order of the relay network can be interpreted and formulated by the well-known max-flow min-cut theorem. Moreover, we extend the analysis to the case of spatially correlated MIMO channels. Approximated explicit expressions for the outage probabilities are obtained in high signal-to-noise ratio regime.
\end{abstract}

Index Terms-Diversity order, Kronecker model, lossy forward, multiple-input multiple-output (MIMO), orthogonal spacetime block code (OSTBC), outage probability

\section{INTRODUCTION}

$\mathbf{I}$ $\mathrm{N}$ the conventional decode-and-forward (DF) relaying protocol [1], [2], the relay forwards the decoded information sequence to its neighboring nodes or the destination only when no errors are detected. A state-of-the-art protocol called lossy forward (LF) was developed in [3], [4], where the relay forwards also erroneous packets or frames. Therefore, better outage performance can be obtained compared to the conventional DF relaying [4].

To the best of the authors' knowledge, the extension of LF concept to the relay network with spatially independent or correlated multiple-input multiple-output (MIMO) channels has not been investigated. Therefore, we consider an LF MIMO transmission system where orthogonal space-time block code (OSTBC) is used for achieving the full diversity order with maximum ratio combining (MRC) in MIMO configurations [5], [6]. It should be noted that the technique to achieve the full diversity order with MRC should not necessarily be restricted to OSTBC when applying the results of this letter and achieving the spatial multiplexing gain is not the aim of this work. The major contributions of the letter are two-fold: (1) Both the transmit and receive correlation of the MIMO channels are taken into consideration for the calculation of outage probability. (2) Approximated explicit expression of the outage probability for the independent MIMO channels, which reveals the diversity order of the OSTBC based MIMO

This work is partially supported by the network compression based wireless cooperative communication systems (NETCOBRA, No. 268209) project, funded by the Academy of Finland.

J. He, V. Tervo, and M. Juntti are with the Centre for Wireless Communications, University of Oulu, Oulu, 90014, Finland (e-mail: jiguang.he@oulu.fi, tervovaltteri@gmail.com, markku.juntti@oulu.fi).

S. Qian and T. Matsumoto are with the school of Information Science, Japan Advanced Institute of Science and Technology (JAIST), Ishikawa, 923 1292, Japan, and the Centre for Wireless Communications, University of Oulu, Oulu, 90014, Finland (e-mail: shen.qian@jaist.ac.jp, matumoto@jaist.ac.jp). relay networks, and that for correlated MIMO channels are obtained in high signal-to-noise ratio (SNR) regime.

Notations: A bold capital letter $\mathbf{A}$ is a matrix, a bold lower case letter $\mathbf{a}$ is a vector, and $a$ is a scalar. We use $\|\mathbf{A}\|_{F}, \mathbf{A}^{H}$, and $\mathbf{A}^{T}$ to denote the Frobenius norm, the complex conjugate transpose, and the transpose of $\mathbf{A}$, respectively. $[\mathbf{a}]_{m}$ is the $m$ th entry of $\mathbf{a}$, and $[\mathbf{A}]_{m n}$ is the $(m, n)$ entry of $\mathbf{A}$. $\operatorname{vec}(\mathbf{A})$ reshapes $\mathbf{A}$ into a vector by stacking A columnwise. $\otimes$ denotes the Kronecker product. $\mathcal{C N}(0,1)$ represents the complex Gaussian distribution where the real and imaginary parts are independent and identically distributed (i.i.d) $\mathcal{N}(0,1 / 2)$, and $\Gamma(\cdot)$ denotes the Gamma function. $a * b=a(1-b)+b(1-a)$ is the binary convolution. $H(\cdot \cdot \cdot)$ and $I(\cdot ; \cdot)$ denote the conditional entropy and the mutual information between the arguments, respectively.

\section{SYSTEM MODEL}

We consider the classical dual-hop MIMO one-way relay network [4], which consists of one source (S), one relay (R), and a single destination (D) equipped with $N_{s}, N_{r}$, and $N_{d}$ antennas, respectively. The three MIMO channels, i.e., source-to-relay (S-R), relay-to-destination (R-D), and sourceto-destination (S-D), regardless of either spatially independent or correlated, are denoted by $\mathbf{H}_{s r}, \mathbf{H}_{r d}$, and $\mathbf{H}_{s d}$, respectively. The probability mass function (pmf) of the binary information sequence $U_{s}$, generated by the source, is $\operatorname{Pr}\left(U_{s}=1\right)=$ $\operatorname{Pr}\left(U_{s}=0\right)=0.5$. The entire transmission round requires two time slots. In the first time slot, the information sequence is generated, encoded, modulated, and broadcast to the relay and the destination. Under the assumption of the LF relaying, the relay decodes, re-encodes, and always forwards the received signal to the destination in the second time slot. At the destination, joint decoding is conducted to recover the data from the source.

The capacity ${ }^{1}$ of the OSTBC based spatially independent point-to-point MIMO channel is expressed as [6]-[8]

$$
\begin{gathered}
C\left(\rho_{i j}\right)=\Theta \log _{2}\left(1+\rho_{i j} \frac{\left\|\mathbf{H}_{i j}\right\|_{F}^{2}}{N_{i} \Theta}\right) \text { bits/s/Hz, } \\
i \in\{s, r\}, j \in\{r, d\}, \text { and } i \neq j,
\end{gathered}
$$

where $\rho_{i j}{ }^{2}$ is the average SNR at the receiver side, $\Theta$ is the information code rate of the OSTBC, and here each entry of $\mathbf{H}_{i j}$ follows $\mathcal{C N}(0,1)$. The squared Frobenius norm of $\mathbf{H}_{i j}$, i.e., $\left\|\mathbf{H}_{i j}\right\|_{F}^{2}$, follows chi-square distribution with $k_{i j}=2 N_{i} N_{j}$ being degrees of freedom. Without loss of

\footnotetext{
${ }^{1}$ We use the term "capacity" in order to make it consistent with the references [6]-[8]. More precisely, the term "capacity" should be replaced by "mutual information" instead.

${ }^{2}$ Note that the subscripts $i$ and $j$ satisfy the following condition: $i \in\{s, r\}$, $j \in\{r, d\}$, and $i \neq j$ throughout the paper.
} 
generality, we set $\Theta=1$ throughout the paper. The distribution of $\gamma_{i j}=\rho_{i j}\left\|\mathbf{H}_{i j}\right\|_{F}^{2} / N_{i}$ can be written as [9]

$$
f\left(\gamma_{i j} ; k_{i j}\right)=\frac{N_{i}\left(\frac{\gamma_{i j} N_{i}}{\rho_{i j}}\right)^{\left(k_{i j} / 2-1\right)} e^{-\frac{\gamma_{i j} N_{i}}{\rho_{i j}}}}{\rho_{i j} \Gamma\left(k_{i j} / 2\right)}, \quad \gamma_{i j} \geq 0 .
$$

Full channel state information (CSI) is assumed to be only available at the receiver side. Each link is assumed to suffer from temporally i.i.d block fading and all the nodes are assumed to be implemented in a half-duplex mode.

\section{Achievable Rate Region and Outage PROBABILITY}

The outage performance analysis can be classified into two distinct cases depending on the decoding outcomes at the relay: It either succeeds or fails in perfectly recovering the information sequence sent from the source.

Due to the transmission orthogonality, the lossy source channel separation theorem ${ }^{3}$ holds for the S-R link [4], [11],

$$
R\left(p_{s r}\right) R_{c, s} \leq C\left(\gamma_{s r}\right),
$$

where $R(\cdot)$ is the rate-distortion function [11], $R_{c, s}$ is the transmission rate of the source, $p_{s r}$ is the Hamming distortion of the S-R link. The Hamming distortion $p_{s r}$ is expressed as [4]

$$
p_{s r}= \begin{cases}H_{b}^{-1}\left[1-\Phi\left(\gamma_{s r}\right)\right], & \text { for } \Phi^{-1}(0) \leq \gamma_{s r} \leq \Phi^{-1}(1), \\ 0, & \text { for } \gamma_{s r} \geq \Phi^{-1}(1),\end{cases}
$$

where $\Phi(x)=C(x) / R_{c, s}=\log _{2}(1+x) / R_{c, s}$ and its inverse function $\Phi^{-1}(x)=2^{R_{c, s} x}-1, H_{b}(x)=-x \log _{2}(x)-(1-$ $x) \log _{2}(1-x), x \in(0,0.5]$ is the binary entropy function and its inverse function $H_{b}^{-1}(\cdot)$ can be found in [4].

\section{A. Case 1: $p_{s r}=0$}

$U_{r}=U_{s}$ with $U_{r}$ representing the estimate of $U_{s}$ at the relay. According to (4), $\gamma_{s r}$ should satisfy the following condition: $\gamma_{s r} \geq \Phi^{-1}(1)$. The achievable rate region for the fully correlated $U_{r}$ and $U_{s}$ is determined by the Slepian-Wolf theorem, i.e., $R_{s} \geq H\left(U_{s} \mid U_{r}\right)=0, R_{r} \geq H\left(U_{r} \mid U_{s}\right)=0$, and $R_{s}+R_{r} \geq H\left(U_{s}, U_{r}\right)=1$, where $R_{s}$ and $R_{r}$ are the source coding rates for the source and relay, respectively.

\section{B. Case 2: $0<p_{s r} \leq 0.5$}

$U_{r} \neq U_{s}$. Based on (4), $\gamma_{s r}$ should satisfy the following condition: $\Phi^{-1}(0) \leq \gamma_{s r} \leq \Phi^{-1}(1)$. A virtual binary symmetric channel (BSC) is utilized to model the relationship between $U_{s}$ and $U_{r}$ with crossover probability $p_{s r}$, i.e., $\operatorname{Pr}\left(U_{r}=1 \mid U_{s}=0\right)=\operatorname{Pr}\left(U_{r}=0 \mid U_{s}=1\right)=p_{s r}$ and $\operatorname{Pr}\left(U_{r}=0 \mid U_{s}=0\right)=\operatorname{Pr}\left(U_{r}=1 \mid U_{s}=1\right)=1-p_{s r}$. It is relatively straightforward to find the pmf of $U_{r}$, which is in the form of $\operatorname{Pr}\left(U_{r}=1\right)=0.5 * p_{s r}=0.5$ and $\operatorname{Pr}\left(U_{r}=0\right)=0.5$.

The achievable rate region for the correlated $U_{r}$ and $U_{s}$ is determined by the theorem of source coding with a helper [11], which is expressed as

$$
\begin{gathered}
R_{s} \geq H\left(U_{s} \mid \hat{U}_{r}\right), \\
R_{r} \geq I\left(U_{r} ; \hat{U}_{r}\right),
\end{gathered}
$$

\footnotetext{
${ }^{3}$ In principle, we should use constellation constrained capacity (CCC) here However, if the instantaneous SNR is low, the Gaussian capacity is almost equal to CCC [10]. If the instantaneous SNR is large, the Hamming distortion would be 0 under the assumption of Gaussian capacity and CCC as long as the fixed transmission rate is less than the CCC. Hence, the use of the binary rate distortion function and the Gaussian capacity is reasonable when using the lossy source channel separation theorem [4].
}

where $\hat{U}_{r}$ is the estimate of $U_{r}$ at the destination.

Similar to (4), the Hamming distortion of the R-D link is in the form of

$$
p_{r d}= \begin{cases}H_{b}^{-1}\left[1-\Phi\left(\gamma_{r d}\right)\right], & \text { for } \Phi^{-1}(0) \leq \gamma_{r d} \leq \Phi^{-1}(1), \\ 0, & \text { for } \gamma_{r d} \geq \Phi^{-1}(1) .\end{cases}
$$

By exploiting (7), (5) and (6) can be further interpreted as

$$
R_{s} \geq \begin{cases}H_{b}\left(p_{s r}\right), & \text { for } R_{r} \geq 1, \\ H_{b}\left(p_{s r} * p_{r d}\right), & \text { for } 0 \leq R_{r} \leq 1 .\end{cases}
$$

\section{Outage Probability}

The lossless source-channel separation theorem holds for the S-D and R-D links. Arbitrarily small error probability can be achieved for these two links [4], if

$$
\begin{aligned}
& R_{s} R_{c, s} \leq C\left(\gamma_{s d}\right), \\
& R_{r} R_{c, r} \leq C\left(\gamma_{r d}\right),
\end{aligned}
$$

where $R_{c, r}{ }^{4}$ is the transmission rate of the relay.

In the outage probability analysis, we consider the equality in (9) and (10). The outage happens when rate pair $\left(R_{s}, R_{r}\right)$ falls outside the achievable rate region. For Case 1, the outage occurs if

$$
\Phi\left(\gamma_{s d}\right)+\Phi\left(\gamma_{r d}\right)<1
$$

According to (8)-(10), the outage happens for Case 2 if

$$
\Phi\left(\gamma_{s d}\right)< \begin{cases}H_{b}\left(p_{s r}\right), & \text { for } \Phi\left(\gamma_{r d}\right) \geq 1 \\ H_{b}\left(p_{s r} * p_{r d}\right), & \text { for } 0 \leq \Phi\left(\gamma_{r d}\right) \leq 1\end{cases}
$$

Since the two cases are independent, the overall outage probability is in the form of

$$
\mathrm{P}_{\text {out }}=\mathrm{P}_{\text {out }}(\text { Case } 1)+\mathrm{P}_{\text {out }}(\text { Case } 2) \text {, }
$$

where $\mathrm{P}_{\text {out }}\left(\right.$ Case 1) and $\mathrm{P}_{\text {out }}($ Case 2) represent the outage probability for Cases 1 and 2, respectively.

The outage probability for Case 1 in (13) can be calculated by taking (11) into consideration along with the precondition $\left\{\gamma_{s r} \geq \Phi^{-1}(1)\right\}$

$$
\begin{gathered}
\mathrm{P}_{\text {out }}(\text { Case } 1)=\int_{\Phi^{-1}(1)}^{\infty} f\left(\gamma_{s r} ; k_{s r}\right) d \gamma_{s r} \int_{\gamma_{s d}=0}^{\Phi^{-1}(1)} \\
\int_{\gamma_{r d}=0}^{\Phi^{-1}\left[1-\Phi\left(\gamma_{s d}\right)\right]} f\left(\gamma_{s d} ; k_{s d}\right) f\left(\gamma_{r d} ; k_{r d}\right) d \gamma_{s d} d \gamma_{r d} .
\end{gathered}
$$

According to (12), the outage probability for Case 2 in (13) can be expressed as

$$
\mathrm{P}_{\text {out }}(\text { Case } 2)=\operatorname{Pr}\left(\mathcal{S}_{1}\right)+\operatorname{Pr}\left(\mathcal{S}_{2}\right) \text {, }
$$

where events $\mathcal{S}_{1}=\left\{0<p_{s r} \leq 0.5, \Phi\left(\gamma_{r d}\right) \geq 1,0 \leq\right.$ $\left.\Phi\left(\gamma_{s d}\right) \leq H_{b}\left(p_{s r}\right)\right\}$ and $\mathcal{S}_{2}=\left\{0<p_{s r} \leq 0.5,0 \leq \Phi\left(\gamma_{r d}\right) \leq\right.$ $\left.1,0 \leq \Phi\left(\gamma_{s d}\right) \leq H_{b}\left(p_{s r} * p_{r d}\right)\right\}$. More specifically, the components in $(1 \overline{5})$ can be further expressed as

$$
\begin{aligned}
& \operatorname{Pr}\left(\mathcal{S}_{1}\right)=\int_{\Phi^{-1}(0)}^{\Phi^{-1}(1)} f\left(\gamma_{s r} ; k_{s r}\right) d \gamma_{s r} \int_{\Phi^{-1}(1)}^{\infty} f\left(\gamma_{r d} ; k_{r d}\right) d \gamma_{r d} \\
& \int_{\Phi^{-1}(0)}^{\Phi^{-1}\left[1-\Phi\left(\gamma_{s r}\right)\right]} f\left(\gamma_{s d} ; k_{s d}\right) d \gamma_{s d} \\
& \operatorname{Pr}\left(\mathcal{S}_{2}\right)= \int_{\Phi^{-1}(0)}^{\Phi^{-1}(1)} f\left(\gamma_{s r} ; k_{s r}\right) d \gamma_{s r} \int_{\Phi^{-1}(0)}^{\Phi^{-1}(1)} f\left(\gamma_{r d} ; k_{r d}\right) d \gamma_{r d} \\
& \int_{\Phi^{-1}(0)}^{\Phi^{-1}\left\{H_{b}\left(\Psi\left(\gamma_{s r}\right) * H_{b}^{-1}\left[1-\Phi\left(\gamma_{r d}\right)\right]\right)\right\}} \\
& f\left(\gamma_{s d} ; k_{s d}\right) d \gamma_{s d}
\end{aligned}
$$

\footnotetext{
${ }^{4} \mathrm{We}$ assume that the transmission rates are the same for all the links, i.e., $R_{c, r}=R_{c, s}=R_{c}$. Therefore, $\Phi(x)$ is also the same for all the links.
} 
where $\Psi\left(\gamma_{s r}\right)=H_{b}^{-1}\left[1-\Phi\left(\gamma_{s r}\right)\right]$.

\section{Spatially Correlated Mimo Channel}

The spatially correlated MIMO channel can be modeled by the Kronecker correlation model [5], [12], [13], which is described as

$$
\mathbf{H}_{i j}=\mathbf{R}_{j}^{1 / 2} \mathbf{H}_{\text {ind }} \mathbf{R}_{i}^{1 / 2},
$$

where $\mathbf{R}_{i}$ and $\mathbf{R}_{j}$ are the deterministic transmit and receive correlation matrices that characterize the spatial correlation among the transmit antenna elements and receive antenna elements, respectively, for MIMO antennas having equal element spacing, $\left[\mathbf{R}_{i}\right]_{m n}=\theta_{i}^{|m-n|}$ and $\left[\mathbf{R}_{j}\right]_{m n}=\theta_{j}^{|m-n|}$ with $\theta_{i}$ and $\theta_{j}$ denoting the transmit and receive correlation coefficients, respectively, and $\mathbf{H}_{\text {ind }}$ is a spatially independent MIMO channel with each entry following $\mathcal{C N}(0,1)$.

The singular value decomposition (SVD) of $\mathbf{R}_{i}$ and $\mathbf{R}_{j}$ is in the form of $\mathbf{R}_{i}=\mathbf{U}_{i} \mathbf{D}_{i} \mathbf{V}_{i}^{H}$ and $\mathbf{R}_{j}=\mathbf{U}_{j} \mathbf{D}_{j} \mathbf{V}_{j}^{H}$, where $\mathbf{U}_{i}$, $\mathbf{V}_{i}, \mathbf{U}_{j}$, and $\mathbf{V}_{j}$ are singular (unitary) matrices, and $\mathbf{D}_{i}$ and $\mathbf{D}_{j}$ are diagonal matrices with singular values on the diagonal. The term $\left\|\mathbf{H}_{i j}\right\|_{F}^{2}$ in (18) is in the form of

$$
\left\|\mathbf{H}_{i j}\right\|_{F}^{2}=\left\|\mathbf{D}_{j}^{1 / 2} \tilde{\mathbf{H}} \mathbf{D}_{i}^{1 / 2}\right\|_{F}^{2}=\sum_{m=1}^{N_{j}} \sum_{n=1}^{N_{i}}\left(\sqrt{\left[\mathbf{D}_{i}\right]_{n n}\left[\mathbf{D}_{j}\right]_{m m}}[\tilde{\mathbf{H}}]_{m n}\right)^{2},
$$

where $\tilde{\mathbf{H}}=\mathbf{V}_{j}^{H} \mathbf{H}_{\text {ind }} \mathbf{U}_{i} . \operatorname{vec}(\tilde{\mathbf{H}})=\left(\mathbf{U}_{i}^{T} \otimes \mathbf{V}_{j}^{H}\right) \operatorname{vec}\left(\mathbf{H}_{\text {ind }}\right)$ with $\mathbf{U}_{i}^{T} \otimes \mathbf{V}_{j}^{H}$ being a unitary matrix. The entries of $\operatorname{vec}(\tilde{\mathbf{H}})$ are also i.i.d. $\mathcal{C N}(0,1)$ because of the properties of the unitary matrix $\mathbf{U}_{i}^{T} \otimes \mathbf{V}_{j}^{H}$.

Remark. Note that we only focus on the scenarios where $\mathbf{R}_{i}$ and $\mathbf{R}_{j}$ are full rank. In other words, the cases where $\theta_{i}=1$ or $\theta_{j}=1$ are excluded.

Lemma 1 [13]-[15]. If $Z_{1}, \cdots, Z_{k}$ are $k$ i.i.d. complex Gaussian random variables with zero mean and variance $\sigma_{m}^{2}$, $m=1,2, \cdots, k, \sigma_{m}^{2} \neq \sigma_{n}^{2}$ if $m \neq n$, then the random variable $X=\sum_{m=1}^{k}\left|Z_{m}\right|^{2}$, which is a sum of independent exponentially distributed random variables, has the following generalized chi-square distribution

$$
f\left(x ; k, \sigma_{1}^{2}, \cdots, \sigma_{k}^{2}\right)=\sum_{m=1}^{k} \frac{\exp \left(-\frac{x}{\sigma_{m}^{2}}\right)}{\sigma_{m}^{2} \prod_{n=1, n \neq m}^{k}\left(1-\frac{\sigma_{n}^{2}}{\sigma_{m}^{2}}\right)} .
$$

According to Lemma 1, $\left\|\mathbf{H}_{i j}\right\|_{F}^{2}$ in (19) is a sum of independent exponentially distributed random variables, and the probability density function (pdf) of $\gamma_{i j}=\rho_{i j}\left\|\mathbf{H}_{i j}\right\|_{F}^{2} / N_{i}$ is given by

$$
\begin{aligned}
& f\left(\gamma_{i j} ; \frac{k_{i j}}{2},\left[\boldsymbol{\sigma}_{i j}\right]_{1}^{2}, \cdots,\left[\boldsymbol{\sigma}_{i j}\right]_{k_{i j} / 2}^{2}\right) \\
& =\frac{N_{i}}{\rho_{i j}} \sum_{m=1}^{k_{i j} / 2} \frac{\exp \left(-\frac{N_{i} \gamma_{i j}}{\rho_{i j}\left[\boldsymbol{\sigma}_{i j}\right]_{m}^{2}}\right)}{\left[\boldsymbol{\sigma}_{i j}\right]_{m}^{2} \prod_{n=1, n \neq m}^{k_{i j} / 2}\left(1-\frac{\left[\boldsymbol{\sigma}_{i j}\right]_{n}^{2}}{\left[\boldsymbol{\sigma}_{i j}\right]_{m}^{2}}\right)},
\end{aligned}
$$

where $\left[\boldsymbol{\sigma}_{i j}\right]_{m}$ is the $m$ th element of $\boldsymbol{\sigma}_{i j}=$ $\left[\sqrt{\left[\mathbf{D}_{j}\right]_{11}\left[\mathbf{D}_{i}\right]_{11}}, \sqrt{\left[\mathbf{D}_{j}\right]_{11}\left[\mathbf{D}_{i}\right]_{22}}, \cdots, \sqrt{\left[\mathbf{D}_{j}\right]_{N_{j} N_{j}}\left[\mathbf{D}_{i}\right]_{N_{i} N_{i}}}\right]^{T}$.

Remark. It is straightforward to extend Lemma 1 to the special case when some of the $Z_{m}$ 's have identical distribution. Combining $Z_{m}$ 's having identical distributions creates a new random variable group, each having different distributions. The elements of the created set of distributions satisfy the precondition of Lemma 1. Then, we can use (20) to calculate the pdf of $X$ with a smaller $k$ number. Similarly, the pdf of $\gamma_{i j}$ can be calculated even if some entries of $\sigma_{i j}$ are identically distributed.

The outage probability for the correlated MIMO case can be calculated in the same manner as that for the independent MIMO case in Section III.

\section{HIGH SNR APPROXIMATION}

We exploit the following three approximations: (1) $\exp (-x) \approx 1-x,(2) \log _{2}(1+x) \approx \frac{x}{\ln (2)},(3) 2^{x}-1 \approx \ln (2) x$ when $|x| \approx 0$. Substituting (2) into (14), (16), and (17), and calculating the integrals using these approximations, the components of the outage probability for the independent MIMO case in (13) can be further approximated as

$\mathrm{P}_{\text {out,ind }}($ Case 1$) \approx \frac{2}{k_{r d}}\left(N_{r}\right)^{\frac{k_{r d}}{2}}\left(N_{s}\right)^{\frac{k_{s d}}{2}}\left[\ln (2) R_{c}\right]^{\frac{k_{s d}+k_{r d}}{2}}$

$\left[\Gamma\left(\frac{k_{s d}}{2}\right) \Gamma\left(\frac{k_{r d}}{2}\right)\right]^{-1}\left[\sum_{m=0}^{k_{r d} / 2}\left(\begin{array}{c}k_{r d} / 2 \\ m\end{array}\right)(-1)^{m} \frac{2}{k_{r d}+2 m}\right] \rho_{s d}^{-\frac{k_{s d}}{2}} \rho_{r d}^{-\frac{k_{r d}}{2}}$

$\mathrm{P}_{\text {out,ind }}($ Case 2$) \approx \frac{2}{k_{s d}}\left[\ln (2) N_{s} R_{c}\right] \frac{k_{s d}+k_{s r}}{2}\left[\Gamma\left(\frac{k_{s d}}{2}\right) \Gamma\left(\frac{k_{s r}}{2}\right)\right]^{-1}$

$\left[\sum_{m=0}^{k_{s d} / 2}\left(\begin{array}{c}k_{s d} / 2 \\ m\end{array}\right)(-1)^{m} \frac{2}{k_{s d}+2 m}\right] \rho_{s d}^{-\frac{k_{s d}}{2}} \rho_{s r}^{-\frac{k_{s r}}{2}}+o\left(\rho_{s d}^{-\frac{k_{s d}}{2}} \rho_{s r}^{-\frac{k_{s r}}{2}}\right)$,

where $o\left(\rho_{s d}^{-\frac{k_{s d}}{2}} \rho_{s r}^{-\frac{k_{s r}}{2}}\right)$ is the higher-order infinitesimal of $\rho_{s d}^{-\frac{k_{s d}}{2}} \rho_{s r}^{-\frac{k_{s r}}{2}}$ as $\rho_{s d}$ and $\rho_{s r}$ approach infinity.

For the spatially correlated MIMO case, the approximations for the outage probability of Cases 1 and 2 are

$$
\begin{aligned}
& \mathrm{P}_{\text {out }, \text { cor }}(\text { Case } 1) \approx \sum_{p=1}^{k_{s d} / 2} \sum_{m=1}^{k_{r d} / 2}\left\{1-\exp \left(-\frac{\ln (2) N_{s} R_{c}}{\rho_{s d}\left[\boldsymbol{\sigma}_{s d}\right]_{p}^{2}}\right)\right. \\
& -\frac{N_{s} \rho_{r d}\left[\boldsymbol{\sigma}_{r d}\right]_{m}^{2}}{N_{r} \rho_{s d}\left[\boldsymbol{\sigma}_{s d}\right]_{p}^{2}-N_{s} \rho_{r d}\left[\boldsymbol{\sigma}_{r d}\right]_{m}^{2}} \exp \left(-\frac{\ln (2) N_{r} R_{c}}{\rho_{r d}\left[\boldsymbol{\sigma}_{r d}\right]_{m}^{2}}\right) \\
& \left.\times\left[\exp \left(\frac{\left(N_{r} \rho_{s d}\left[\boldsymbol{\sigma}_{s d}\right]_{p}^{2}-N_{s} \rho_{r d}\left[\boldsymbol{\sigma}_{r d}\right]_{m}^{2}\right) \ln (2) R_{c}}{\rho_{s d} \rho_{r d}\left[\boldsymbol{\sigma}_{s d}\right]_{p}^{2}\left[\boldsymbol{\sigma}_{r d}\right]_{m}^{2}}\right)-1\right]\right\} \\
& /\left\{\prod_{q=1, q \neq p}^{k_{s d} / 2}\left(1-\frac{\left[\boldsymbol{\sigma}_{s d}\right]_{q}^{2}}{\left[\boldsymbol{\sigma}_{s d}\right]_{p}^{2}}\right) \prod_{n=1, n \neq m}^{k_{r d} / 2}\left(1-\frac{\left[\boldsymbol{\sigma}_{r d}\right]_{n}^{2}}{\left[\boldsymbol{\sigma}_{r d}\right]_{m}^{2}}\right)\right\}, \\
& \mathrm{P}_{\text {out }, \text { or }}(\text { Case } 2) \approx \sum_{p=1}^{k_{s r} / 2} \sum_{m=1}^{k_{s d} / 2}\left\{1-\exp \left(-\frac{\ln (2) N_{s} R_{c}}{\rho_{s r}\left[\boldsymbol{\sigma}_{s r}\right]_{p}^{2}}\right)\right. \\
& -\frac{\rho_{s d}\left[\boldsymbol{\sigma}_{s d}\right]_{m}^{2}}{\rho_{s r}\left[\boldsymbol{\sigma}_{s r}\right]_{p}^{2}-\rho_{s d}\left[\boldsymbol{\sigma}_{s d}\right]_{m}^{2}} \exp \left(-\frac{\ln (2) N_{s} R_{c}}{\rho_{s d}\left[\boldsymbol{\sigma}_{s d}\right]_{m}^{2}}\right) \\
& \left.\times\left[\exp \left(\frac{\left(\rho_{s r}\left[\boldsymbol{\sigma}_{s r}\right]_{p}^{2}-\rho_{s d}\left[\boldsymbol{\sigma}_{s d}\right]_{m}^{2}\right) \ln (2) N_{s} R_{c}}{\rho_{s d} \rho_{s r}\left[\boldsymbol{\sigma}_{s r}\right]_{p}^{2}\left[\boldsymbol{\sigma}_{s d}\right]_{m}^{2}}\right)-1\right]\right\} \\
& /\left\{\prod_{q=1, q \neq p}^{k_{s r} / 2}\left(1-\frac{\left[\boldsymbol{\sigma}_{s r}\right]_{q}^{2}}{\left[\boldsymbol{\sigma}_{s r}\right]_{p}^{2}}\right) \prod_{n=1, n \neq m}^{k_{s d} / 2}\left(1-\frac{\left[\boldsymbol{\sigma}_{s d}\right]_{n}^{2}}{\left[\boldsymbol{\sigma}_{s d}\right]_{m}^{2}}\right)\right\} .
\end{aligned}
$$

\section{A. Diversity Order and Spatial Correlation}

The diversity orders of the S-R, R-D, and S-D links are $N_{s} N_{r}, N_{r} N_{d}$, and $N_{s} N_{d}$, respectively. Referring to the summation of (22) and (23), the detailed expression for the diversity order $d$ is in the form of

$$
d=N_{s} N_{d}+\min \left\{N_{s} N_{r}, N_{r} N_{d}\right\} .
$$




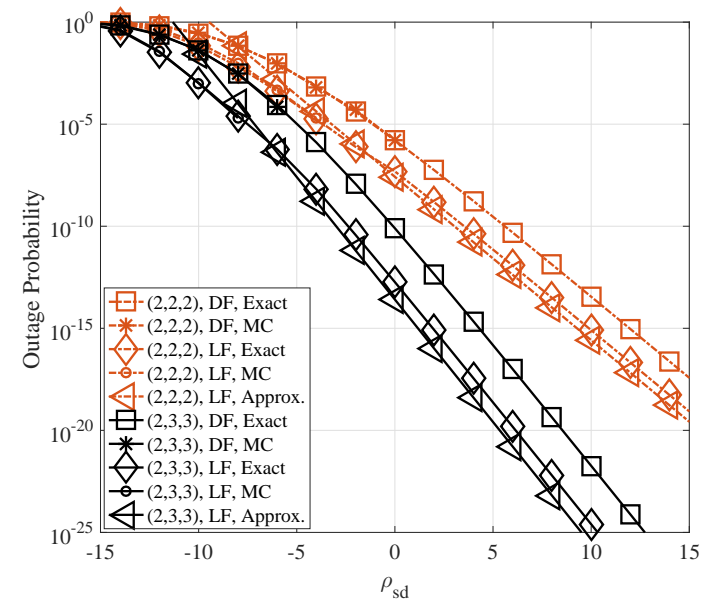

Fig. 1. Diversity order of the outage probability of the spatially independent MIMO relay network.

The diversity order of the relay network is formulated by the max-flow min-cut theorem [16], which can be applied to more general MIMO multi-source multi-relay networks.

Due to the existence of the spatial correlation, the capacity of each link reduces accordingly. Therefore, the outage performance decreases as the increase of the correlation coefficients.

\section{Simulation Results}

In this section, we provide a bunch of experiments to verify our theoretical derivations. The conventional DF is offered as a benchmark scheme. For all the simulations, we set $\rho_{s r}=\rho_{r d}=\rho_{s d}+5 \mathrm{~dB}$ and $R_{c, s}=R_{c, r}=0.5$. In the first experiment, we set different values for the number of antennas, e.g., $\left(N_{s}, N_{r}, N_{d}\right)=\{(2,2,2),(2,3,3)\}$. The simulation results (including Monte-Carlo (MC) simulation results) for the spatially independent MIMO case are provided in Fig. 1. It can be observed that the diversity order of the LF relay network follows (26). The MC simulation results are closely matched with the theoretical curves. In the second experiment, we take into account the correlation of the MIMO channels while setting $\left(N_{s}, N_{r}, N_{d}\right)=(2,2,2)$. Different setups for the correlation coefficients, e.g., $\left(\theta_{s}, \theta_{r}, \theta_{d}\right)=$ $\{(0.9,0.93,0.95),(0.8,0.7,0.75)\}$, are taken into consideration. The simulation results, including the results for the spatially independent MIMO case (i.e., $\left.\left(\theta_{s}, \theta_{r}, \theta_{d}\right)=(0,0,0)\right)$, are provided in Fig. 2. The diversity order of the correlated MIMO case is the same as that of independent MIMO case owing to the full rank of the correlation matrices.

\section{CONCLUSION}

We studied the impact of spatial correlation of the MIMO channel on the outage probability of the relay networks using OSTBC, where the LF relaying strategy is applied. The explicit approximations of the outage probabilities were obtained using high SNR approximation. The diversity order has been interpreted and formulated by the classical max-flow min-cut theorem, which is applicable to more general MIMO multi-source multi-relay networks. Spatial correlation does not

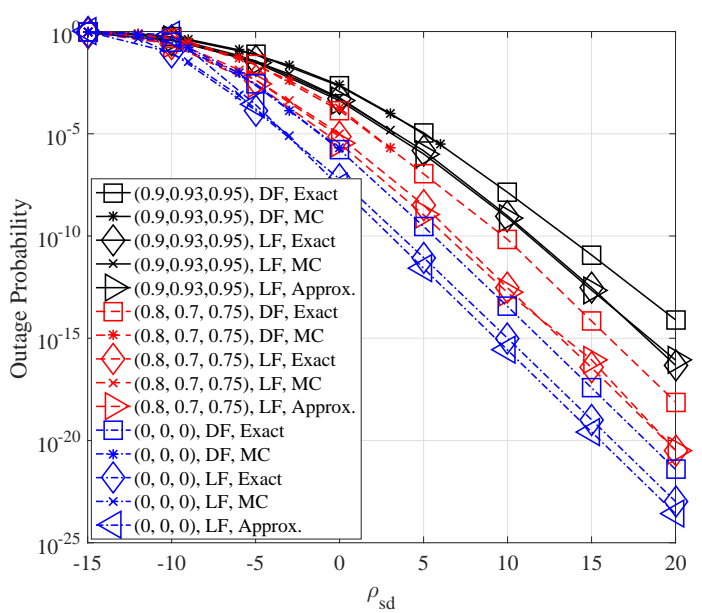

Fig. 2. Comparison between spatially independent and correlated MIMO relay networks.

change the diversity order as long as correlation matrices are full rank.

\section{REFERENCES}

[1] J. N. Laneman and G. W. Wornell, "Distributed space-time-coded protocols for exploiting cooperative diversity in wireless networks," IEEE Trans. Inf. Theory, vol. 49, no. 10, pp. 2415-2425, Oct 2003.

[2] J. N. Laneman, D. N. C. Tse, and G. W. Wornell, "Cooperative diversity in wireless networks: Efficient protocols and outage behavior," IEEE Trans. Inf. Theory, vol. 50, no. 12, pp. 3062-3080, Dec 2004.

[3] K. Anwar and T. Matsumoto, "Accumulator-assisted distributed turbo codes for relay systems exploiting source-relay correlation," IEEE Commun. Lett., vol. 16, no. 7, pp. 1114-1117, July 2012.

[4] X. Zhou, M. Cheng, X. He, and T. Matsumoto, "Exact and approximated outage probability analyses for decode-and-forward relaying system allowing intra-link errors," IEEE Trans. Wireless Commun., vol. 13, no. 12, pp. 7062-7071, Dec 2014.

[5] A. Maaref and S. Aissa, "Performance analysis of orthogonal space-time block codes in spatially correlated MIMO Nakagami fading channels," IEEE Trans. Wireless Commun., vol. 5, no. 4, pp. 807-817, Apr. 2006.

[6] B. K. Chalise and L. Vandendorpe, "Outage probability analysis of a MIMO relay channel with orthogonal space-time block codes," IEEE Commun. Lett., vol. 12, no. 4, pp. 280-282, Apr. 2008.

[7] S. Sandhu and A. Paulraj, "Space-time block codes: a capacity perspective," IEEE Commun. Lett., vol. 4, no. 12, pp. 384-386, Dec 2000.

[8] J. Perez, J. Ibanez, L. Vielva, and I. Santamaria, "Closed-form approximation for the outage capacity of orthogonal STBC," IEEE Commun. Lett., vol. 9, no. 11, pp. 961-963, Nov 2005.

[9] J. Cui and A. U. H. Sheikh, "Outage probability of cellular radio systems using maximal ratio combining in the presence of multiple interferers," IEEE Trans. Commun., vol. 47, no. 8, pp. 1121-1124, Aug 1999.

[10] G. Ungerboeck, "Channel coding with multilevel/phase signals," IEEE Trans. Inf. Theory, vol. 28, no. 1, pp. 55-67, Jan 1982.

[11] A. E. Gamal and Y.-H. Kim, Network Information Theory. New York, NY, USA: Cambridge University Press, 2012.

[12] A. M. Tulino, A. Lozano, and S. Verdu, "Impact of antenna correlation on the capacity of multiantenna channels," IEEE Trans. Inf. Theory, vol. 51, no. 7, pp. 2491-2509, Jul. 2005.

[13] B. K. Chalise, "On the performance of SR and FR protocols for OSTBCbased AF-MIMO relay system with channel and noise correlations,' IEEE Trans. Veh. Technol., vol. 65, no. 8, pp. 5959-5971, Aug 2016.

[14] A. Bletsas, H. Shin, and M. Z. Win, "Cooperative communications with outage-optimal opportunistic relaying," IEEE Trans. Wireless Commun., vol. 6, no. 9, pp. 3450-3460, September 2007.

[15] D. Hammarwall, M. Bengtsson, and B. Ottersten, "Acquiring partial CSI for spatially selective transmission by instantaneous channel norm feedback," IEEE Trans. Signal Process., vol. 56, no. 3, pp. 1188-1204, Mar. 2008.

[16] S. Y. R. Li, R. W. Yeung, and N. Cai, "Linear network coding," IEEE Trans. Inf. Theory, vol. 49, no. 2, pp. 371-381, Feb 2003. 